\title{
CARACTERÍSTICAS DE LOS JUEGOS TRADICIONALES DE LA COMUNIDAD EMBERA CHAMÍ DE PUEBLO RICO, RISARALDA ${ }^{1}$
}

\author{
CHARACTERISTICS OF THE TRADITIONAL GAMES OF THE EMBERA \\ CHAMÍ COMMUNITY OF PUEBLO RICO, RISARALDA
}

\author{
CARACTERÍSTICAS DOS JOGOS TRADICIONAIS DA COMUNIDADE \\ EMBERA CHAMÍ DE PUEBLO RICO, RISARALDA
}

Edwin Giraldo Duque
Yenni Galeano López

Resumen

Este artículo presenta una investigación cuyo objetivo fue describir los juegos tradicionales de la comunidad embera chamí en los resguardos de Iumade, Kemberde y Santa Teresa, ubicados en Pueblo Rico, Risaralda, Colombia. Se desarrolló a partir del método cualitativo con un diseño fenomenológico. La muestra se conformó a partir de 30 participantes pertenecientes a la comunidad embera chamí de los tres resguardos, a quienes se les aplicó una entrevista semiestructurada. Las preguntas se diseñaron a partir de las categorías que surgieron de la revisión de las unidades de análisis contenidas en los diarios de campo sobre la identificación de los juegos en la comunidad. Entre los resultados más relevantes, se encuentra que los juegos estudiados tienen su origen en la subsistencia como comunidad, ya que originalmente se utilizaron para la caza y las guerras contra otros pueblos. Se concluye que los juegos han tenido una disminución en su ejecución en las nuevas generaciones, las cuales prefieren practicar deportes de origen externo a la comunidad como lo es el fútbol.

Palabras clave: cultura; tradiciones indígenas; deportes indígenas; costumbres indígenas; cultura; deporte

\section{Abstract}

This article presents a research aimed at describing the traditional games of the Embera Chamí community in the Iumade, Kemberde and Santa Teresa people, located in Pueblo Rico, Risaralda, Colombia. It was developed from the qualitative method with a phenomenological design. The sample was formed by 30 participants belonging to the Embera Chamí community of the three towns, to whom a semi-structured interview was applied. The questions were designed based on the categories that emerged from the review of the units of analysis contained in the field diaries on the identification of games in the community. Among the most relevant results is that the games studied have their origin in subsistence as a community, since originally they were used for hunting and wars against other peoples. It is concluded that the games have had a decrease in reproduction in the new generations, which prefer to play sports of external origin to the community such as soccer.

Key words: culture; indigenous traditions; indigenous sports; indigenous customs; culture; sports

1 Proyecto financiado por el Sistema de Investigación, Desarrollo Tecnológico e Innovación del sENA (SENNOvA), Centro de Comercio y Servicios, Regional Risaralda.

2 Licenciado en Educación Física, Instructor del SENA, Bogotá, Colombia. Correo electrónico: edgiraldod@misena.edu.co. Orcid: 0000-0002-4973-940X

3 Especialista en gestión del talento humano. Instructora sENA, Bogotá, Colombia. Correo electrónico: ygaleanol@sena.edu.co. Orcid: 0000-0002-4034-9878 


\section{Resumo}

Este artigo apresenta uma investigação cujo objetivo foi descrever os jogos tradicionais da comunidade Embera Chamí nas reservas de Iumade, Kemberde e Santa Teresa, localizadas em Pueblo Rico, Risaralda, Colômbia. Foi desenvolvido a partir do método qualitativo, com desenho fenomenológico. A amostra foi composta por 30 participantes pertencentes à comunidade Embera Chamí das três reservas, aos quais foi aplicada uma entrevista semiestruturada. As questões foram elaboradas com base nas categorias que emergiram da revisão das unidades de análise contidas nos diários de campo sobre a identificação de jogos na comunidade. Entre os resultados mais relevantes, verifica-se que os jogos estudados têm origem na subsistência como comunidade, uma vez que foram originalmente utilizados para caça e guerras contra outros povos. Conclui-se que os jogos tiveram uma diminuição de sua execução nas novas gerações, que preferem praticar esportes de origem externa à comunidade, como o futebol.

Palavras-chave: cultura; tradições indígenas; esporte indígena; costumes indígenas; cultura; esporte

Para citar este artículo:

Galeano Lopez, Y., y Giraldo Duque, E. (2019). Características de los juegos tradicionales de la comunidad Embera Chamí de Pueblo Rico, Risaralda. Lúdica Pedagógica, 30, 51-58. https://doi.org/10.17227/ludica. num30-11125 


\section{INTRODUCCIÓN}

En la actualidad, el campo de la investigación en pueblos indígenas en Colombia ha ido en auge y se pueden encontrar estudios sobre etnoeducación, especialmente enfocados en las matemáticas y en las experiencias culturales de estos pueblos y su interacción con otras comunidades (Tamayo y Cuellar 2016; Aroca, 2010; Aroca 2013). Este crecimiento puede deberse a varias causas, una de ellas es el fin que tiene la etnoeducación para Boadnar (citado por Rojas y Castillo, 2005): busca un reconocimiento y respeto por las culturas autóctonas. Otra explicación del interés de la academia por los pueblos indígenas es el intercambio de conocimiento que se puede lograr con ellos.

De igual manera, todos estos estudios permiten una revitalización de la identidad indígena, tal como lo menciona Mavisoy (2014) llegando a proponer una integración entre los conocimientos exógenos (Academia) y los endógenos (culturas indígenas) para desarrollos de nuevos contextos de estudio. Para lograr esto, es válido enfocar la atención en las costumbres y actividades propias de los pueblos indígenas, para el caso de sus juegos tradicionales. Estos juegos, para Castañer y Trigo (1998), forman parte de la riqueza cultural como comunidad de la zona donde se practica.

A nivel académico, Moreno (2008) hace una referencia de actividades y trabajos que se han realizado sobre los juegos tradicionales en donde se destacan eventos de orden nacional, la caracterización de una comunidad embera con respecto a sus prácticas recreativas y deportivas hasta llegar al momento en donde el juego del turmequé pasa de ser un juego indígena a convertirse en el deporte nacional de Colombia por resolución del Congreso en el año 2000. De igual manera, el Ministerio de Educación Nacional (2015) brinda una cartilla sobre los juegos infantiles y artes tradicionales del pueblo embera katío del Alto Sinú.

El pensar en temas como los juegos tradicionales, sobre todo en población indígena, como se ha planteado, ayuda a darle valor a su cultura, que se ha ido perdiendo en los pueblos aborígenes, en especial en los embera chamí, ubicados en el departamento de Risaralda, tal como lo menciona el Ministerio de Cultura (2010): “Otros problemas se relacionan con el contacto permanente de los chamí con la cultura occidental, que ha generado una pérdida considerable del conocimiento, usos y costumbres propias" (p. 8).

Es por esto que se propuso identificar las características y los alcances de los juegos tradicionales de la comunidad indígena embera chamí ubicada en el municipio de Pueblo Rico, Risaralda, planteándose el siguiente interrogante: ¿cuáles son las características y alcances de los juegos tradicionales de la comunidad embera chamí en los resguardos de Iumade, Kemberde y Santa Teresa, ubicados en Pueblo Rico, Risaralda, Colombia?

\section{METOdología}

Tipo

El presente estudio es de tipo cualitativo, el cual, según Hernández, Fernández y Baptista (2014), “se enfoca en comprender los fenómenos, explorándolos desde la perspectiva de los participantes en un ambiente natural y en relación con su contexto" (p. 358). Para el caso, se exploraron los juegos tradicionales de los embera chamí ubicados en Pueblo Rico, Risaralda. El propósito es comprender las características de tipo cultural, ancestral, y técnicas de estas actividades para los embera.

\section{Diseño y participantes}

El diseño utilizado es el fenomenológico, el cual busca entender la experiencia de personas sobre un fenómeno o múltiples perspectivas de este. Para el caso, el fenómeno fue los juegos tradicionales y sus alcances dentro de la comunidad (Hernández et al., 2014). El estudio se desarrolló en los resguardos de Iumade, Santa Teresa, Kemberde, pertenecientes al municipio de Pueblo Rico, Risaralda. Se entrevistaron 30 personas que hacían parte de los mencionados resguardos. El criterio de inclusión se basó en ser personas que pertenecieran a la comunidad indígena, que presentaran el consentimiento informado y que fueran mayores de edad.

\section{Instrumento y procedimiento}

Los instrumentos utilizados fueron diarios de campo y entrevista. Los diarios de campo se utilizaron en primera medida en los tres resguardos cuando la comunidad disfrutaba de su ocio, o cuando se les 
solicitaba mostraran cuáles eran sus juegos tradicionales, en estas observaciones las participaciones de los investigadores eran moderadas. Luego, se pasó a la elaboración de una entrevista semiestructurada basada en las unidades de análisis. Dichas entrevistas fueron aplicadas a 30 sujetos de manera individual. En la tabla 1 se muestra las unidades de análisis que sirvieron de referencia para la elaboración de la entrevista.

Tabla 1. Unidades de análisis

\begin{tabular}{|c|c|c|c|}
\hline Objetivo específico & Instrumento & Objetivo del instrumento & Unidades de análisis \\
\hline $\begin{array}{l}\text { Determinar los orígenes de los juegos } \\
\text { tradicionales de la comunidad embera } \\
\text { chamí del resguardo de Iumade, } \\
\text { Kemberde y Santa Teresa, ubicados en } \\
\text { Pueblo Rico, Risaralda, Colombia. }\end{array}$ & Entrevista & $\begin{array}{l}\text { Revelar las razones por } \\
\text { las cuales nacieron los } \\
\text { juegos tradicionales en las } \\
\text { comunidades estudiadas. }\end{array}$ & $\begin{array}{l}\text { - Subsistencia } \\
\text { - Recreación }\end{array}$ \\
\hline $\begin{array}{l}\text { Identificar las características de los } \\
\text { juegos tradicionales de la comunidad } \\
\text { embera con respecto a su técnica, } \\
\text { reglamento, y competencia. }\end{array}$ & Entrevista & $\begin{array}{l}\text { Mostrar cómo se puede } \\
\text { poner en práctica los } \\
\text { juegos tradicionales. }\end{array}$ & $\begin{array}{l}\text { - Técnica } \\
\text { - Reglamento }\end{array}$ \\
\hline $\begin{array}{l}\text { Examinar los alcances de los juegos } \\
\text { tradicionales para la cultura de la comunidad } \\
\text { de Iumade, Kemberde y Santa Teresa, ubicados } \\
\text { en Pueblo Rico, Risaralda, Colombia. }\end{array}$ & Entrevista & $\begin{array}{l}\text { Evidenciar cómo los } \\
\text { juegos le han aportado a la } \\
\text { comunidad en su historia. }\end{array}$ & $\begin{array}{l}\text { - } \text { Subsistencia } \\
\text { - Relaciones con otras } \\
\text { comunidades } \\
\text { - Enseñar a las nuevas } \\
\text { generaciones } \\
\text { - Percepción del juego } \\
\text { en la comunidad }\end{array}$ \\
\hline
\end{tabular}

Fuente: elaboración propia a partir de orientar las unidades de análisis a cada objetivo del proyecto.

Seguido a esto, se realizó una preparación de los datos transcribiéndolos al programa de Word, y desde allí se ejecutó la revisión de estos y su relación con las unidades de análisis planteadas inicialmente. De esta forma, emergieron unas categorías con sus respectivos códigos (Hernández et al., 2014). Por último, se relacionaron dichas categorías con su posterior definición, buscando generar explicaciones sobre los juegos tradicionales de la comunidad embera chamí en los resguardos analizados.

A continuación, se presentan los resultados de la investigación. Para este proceso se tomaron las palabras en contexto de algunos de los sujetos que participaron del estudio. Con el propósito de reservar la identidad de los participantes, de aquí en adelante se identificarán a los sujetos con la letra S seguida de un número (ejemplo: S1, S2, etc.).

Los juegos tradicionales de la comunidad embera chamí, localizada sobre el río San Juan, en el munici- pio de Pueblo Rico, tienen un origen enfocado en la subsistencia como comunidad. Para los habitantes, estos juegos "existen hace muchos años, son ancestrales... la trampa es un juego que salió de la cacería. Los juegos son la tradición de nosotros...” (S1). "Los juegos los practican en los eventos" (S8).

Es así que los elementos de los juegos como la cerbatana, el arco con flecha, la trampa y la lanza en un principio fueron empleados para la cacería de animales y para las guerras contra otras comunidades. Esta idea compagina con lo publicado por Ulloa (1992) y Ministerio de Cultura (s. f.), en donde después de un arduo trabajo académico e investigativo, se hace una caracterización de esta población, indicando que estos desde un principio han estado sometidos a procesos de conquista y colonización, razón por la cual la guerra ha sido parte de la historia de ellos. Es de esta manera que, seguramente, elementos como la cerbatana y el arco con flecha han tenido lugar en la historia de la comunidad chamí. De igual forma, estos conflictos han podido ocasionar la modificación de la cultura embera: "En esta dinámica han transcurrido 
cinco siglos de historia de explotación, dominio y violencia hacia los indígenas, modificándoles tanto el espacio vital como sus elementos culturales" (Ulloa, 1992, p. 88).

Retomando esta idea, los juegos hoy existentes pueden ser considerados parte de la cultura y de la historia de los embera chamí que les han permitido subsistir. Esta subsistencia también es característica de los embera acentuados sobre el alto San Juan, en donde su mayor empleabilidad ha estado enfocada hacia cultivos de plátano, maíz, café, cacao, caña y arroz. Esto, acompañado de la cacería: "Como los cultivos no requieren de excesivo trabajo, sino de deshierbes y limpieza esporádica, los hombres salen de caza y pesca" (Ulloa, 1992, p. 94).

Con respecto a los juegos, en la actualidad, estos hacen parte de la cultura de los embera y son utilizados en su gran mayoría para la recreación y muestras artísticas entre pueblos, tal como lo expresa los sujetos participes del estudio: "El juego es para la recreación... Yo juego cada dos meses, cuando se hacen los eventos" (S3).

Se puede decir que no es tan claro cuánto tiempo la comunidad dedica a los juegos en los resguardos de Pueblo Rico; de igual manera, se nota desconocimiento sobre la empleabilidad de los juegos por parte de las nuevas generaciones $\mathrm{y}$, como se ha dicho, estas actividades son más practicadas cuando existe la necesidad de mostrar su cultura a otros pueblos.

Una posible explicación a lo encontrado sobre el alejamiento de las nuevas generaciones y los juegos la formula el Ministerio de Cultura (2010), que indica que el pueblo embera tiende a perder su cultura y a darle mayor prioridad a culturas de otros pueblos, lo cual apoya la Sentencia T025 emitida en 2004 por la Suprema Corte Constitucional. Esto puede ser evidenciado a través de la observación de los diarios de campo de la presente investigación, en donde gran parte del tiempo de ocio de los sujetos en los resguardos es empleado en jugar el deporte del fútbol y muy pocas veces en practicar sus juegos tradicionales. De igual manera, esta idea es apoyada por algunos emberas entrevistados cuando expresan: "Yo casi no practico los juegos de mi comunidad... juego es fútbol" (S1).
Otro apoyo a esta idea es la que plantea Carreño (2003): "Pudieron establecer que la mayor parte de las actividades que los embera enmarcan en el término juego están determinados por culturas, foráneas" (p. 37). Algo similar a lo que sucedió en la presente investigación.

Por otra parte, se ha encontrado que los juegos tradicionales son enseñados por parte de los mayores $\mathrm{y}$ algunas veces son los maestros los encargados de enseñarlos en la escuela: "Mis papás me enseñaron a jugar..." (S2); "en la escuela enseñan a jugar" (S8); "los mayores le enseñan a jugar a los niños... Bien, porque siempre hay que dejarles enseñanza a los niños que están tras de nosotros y no debemos permitir que los juegos se acaben" (S6).

El fin que se busca con esta enseñanza es pasar los conocimientos y dominios culturales a las nuevas generaciones de emberas. Para lograr este fin, se necesita una identidad cultural promovida desde la educación, es decir, una etnoeducación que vincule los estándares nacionales y la cultura indígena, tal como lo han propuesto en diferentes áreas (Aroca, 2010; Aroca, 2013; Sánchez, Aguirre y Ochoa, 2015; Tamayo y Cuellar, 2016), buscando de esta forma una identidad cultural como signo de perpetuar las costumbres y las tradiciones. Con esto no se quiere decir que el tipo de educación brindada en los resguardos de Pueblo Rico no promueva la identidad cultural, solo se quiere dejar destacado que la educación es el camino para llegar a tales fines, ayudando en la conservación de las costumbres y tradiciones de la cultura embera.

La familia es otra de las instituciones que se destaca a la hora de la enseñanza de los juegos. La familia para la vida embera es muy importante y brinda la estructura social, que radica en unidades familiares como base de su sociedad (Ulloa, 1992). Es así que se pueden considerar los juegos tradicionales como actividades que permiten fortalecer esa familia del pueblo embera chamí localizado en los resguardos de Pueblo Rico, Risaralda.

Con respecto a la participación de las mujeres en los juegos tradicionales, se pudo detectar que esta es muy baja. La práctica de las mujeres en los juegos puede darse únicamente por la necesidad de aprenderlos para enseñarlos a las nuevas generaciones. 
"Las mujeres deben de aprender para enseñarles a los niños..." (S6); "algunas juegan, pero hay muy poquito interés" (S9).

Ellas, a través de la historia, han desarrollado otras actividades como es la cestería, las artesanías de collares, las danzas, entre otras. Tal como lo indica una mujer embera participante de la muestra.

"A mí me enseñaron del juego sobre el barro a diferencia de los hombres que juegan trampa. Las mujeres en el barro, canastas y collares" (S6).

Sobre estas prácticas Ulloa (1992) y Vasco (1987) describen los alcances de la cestería (elaboración de canastos) en los resguardos embera y algunos de sus fines. La cestería es una actividad practicada por todas las mujeres de la comunidad, la cestería chamí se distingue por ser la más variada y rica entre todos los pueblos indígenas colombianos. Dependiendo del material se elaboran diferentes tipos de canastos. De la iraca, hoja blanca, hinguru y joro, se cortan los tallos más largos, para separarles la corteza, la cual se adelgaza hasta convertirla en fibra textil. Con ellas se tejen los diferentes canastos (jabara) elaborados para guardar la harina de maíz y el arroz.

Cabe decir que, para identificar más sobre las actividades de las mujeres en los resguardos de Pueblo Rico y sus alcances, se hace necesario una extensión del tema de la presente idea de investigación, para poder tener mayor claridad sobre esa categoría, ya que los alcances de la presente investigación no estuvieron enfocados en ese punto.

Por último, se expondrán los juegos tradicionales identificados y sus características en lo que respecta a sus reseñas y sus empleabilidades dentro de los resguardos analizados. Los juegos son el arco con flecha, la cerbatana, la trampa y la lanza.

El arco con flecha tiene su nacimiento en la búsqueda de la supervivencia de la comunidad, este ha sido utilizado para defender el territorio y ha servido en la cacería de animales.

"El arco con flecha nació en el campo para conocer de este juego para cazar" (S5).

Ulloa (1992) relata que este elemento es utilizado por los embera en general para cazar pescados cuando el cauce del río aumenta.
El juego consiste en insertar una flecha en una base que lo permita, ubicada a una distancia aproximada de 4 metros. Para el desarrollo de este juego, se necesita un arco hecho normalmente de guadua, resortes y flechas artesanales fabricadas por la misma comunidad.

Del juego de la cerbatana se pudo identificar que tiene su origen en la necesidad de conseguir alimentos. Este era utilizado en un principio para cazar, tenía como particularidad el uso del veneno de rana en la punta de las flechas para conseguir neutralizar la presa.

"La cerbatana nació en el resguardo para cazar" (S6).

Sobre las características de este juego, se puede indicar que es similar al encontrado en el pueblo Embera Katio localizado en el alto del Sinú, publicado por el Ministerio de Educación Nacional (2015), en esta presentación describen el juego de la siguiente manera:

Consiste en lanzar un dardo con el boroco o cerbatana hacia el blanco; este blanco se encuentra aproximadamente a unos treinta metros de distancia. El jugador que logre alcanzar el objetivo dando en el blanco gana. Si ambos dan en el blanco, se repite la acción hasta que alguno falle (p. 47).

Esta definición es algo similar a la forma de ejecutarse el juego en los resguardos de Pueblo Rico estudiados, en donde se intenta insertar una flecha que es decorada con algodón y plumas. Esta es enviada a través de una cerbatana empujada por medio del aire exhalado por la boca.

Los elementos que se necesitan para su práctica son una cerbatana hecha normalmente de palos de chontaduros (palma perteneciente a la familia de plantas arecáseas), una macana (forro natural de colmena), "brea" flechas (palo de guadua) y el tarro hecho en guadua para cargar las flechas.

Otro de los juegos es la trampa, la cual "en un principio debía atrapar presas" (S2).

El juego de la trampa se asocia a la cacería, tal como lo relata el sujeto entrevistado. Esta actividad tiene dos momentos: empieza cuando cada participante debe de armar su trampa, y después debe de realizar lanzamientos de un objeto (palo o piedra) a la cabeza de esta para que se active y así poder ganar puntaje. 
Todos los elementos utilizados son de orden natural y van desde pedazos de guarda, bejuco, y machete.

La lanza, por su parte, como todos los juegos encontrados, tiene su génesis en la supervivencia. Con esta se podía combatir contra otra persona o presa, tal como lo menciona uno de los embera chamí.

"Inicialmente consistía en pegarle a un animal para comerlo" (S22).

En la actualidad la empleabilidad de este instrumento a manera de juego tiene algo de similitud con la prueba de atletismo de jabalina. El objetivo es hacer un lanzamiento de dicho elemento buscando su caída de forma correcta (enterrada) y alcanzar una mayor distancia que la de sus adversarios. En los resguardos analizados, este elemento es hecho de guadua y se juega en una zona verde, como lo son sus canchas de fútbol.

\section{CONCLUSIONES}

- Según los resultados encontrados, los juegos tradicionales de la comunidad embera de Pueblo Rico, Risaralda, tienen su origen en la subsistencia como comunidad, en donde los juegos y sus elementos eran utilizados como prácticas para conseguir alimentos o para defender su territorio.

- Los juegos que se identificaron en el presente estudio en los resguardos seleccionados carecen de un reglamento y técnicas de práctica claras, debido a que tales juegos en la actualidad no son practicados permanentemente. Se sugiere más estudios en este tipo de pueblos indígenas, en donde se pueda medir las motivaciones y el sentido de pertenecía de las nuevas generaciones con la cultura indígena. Además de cuál es el grado de influencia en la actualidad de otras culturas en los jóvenes embera.

- Según los juegos hallados, se concluye que estos son propios de los embera chamí del departamento de Risaralda, y que estos le dan una autenticidad de orden cultural a dicha comunidad, permitiendo la unión de las familias y de las nuevas generaciones con sus ancestros a la hora de socializar dichas prác- ticas. Se recomienda un estudio en donde, además de los juegos, se tengan en cuenta actividades como bailes y elaboración de artesanías propias de esta comunidad que permitan profundizar en los valores lúdicos y culturales que tienen como pueblo, las cuales quizás sean más practicadas en la actualidad.

- Producto de esta investigación se diseñó una cartilla que contiene las características de los juegos tradicionales de la comunidad embera chamí que se encontraron en los resguardos anteriormente mencionados. En este documento, se pueden encontrar una descripción más amplia de las actividades dentro de los resguardos, además se presentan ilustraciones y fotografías al respecto. Tal documento es de orden público y puede ser consultado en el repositorio de la biblioteca del SENA.

\section{REFERENCIAS}

Aroca, A. (2010). Una experiencia de formación docente en Etnomatemáticas: estudiantes afrodescendientes del Puerto de Buenaventura, Colombia. Revista Horizonte, 28(1), 87-95.

Aroca, A. (2013). Los escenarios de exploración en el Programa de Investigación en Etno-matemáticas. Educación matemática, 25(1), 111-131.

Carreño, J. (2003). El cuerpo y el juego en los embera de Tumburrulá. Revista Lúdica Pedagógica, 1(8), 33-39.

Castañer, M., y Trigo, E. (1998). Globalidad e interdisciplina curricular en la enseñanza primaria. Barcelona: Inde.

Corte Constitucional de Colombia. (26 de enero de 2009). Sentencia T-025. (Manuel José Cepeda Espinosa, M. P.).http://www.corteconstitucional.gov.co/relatoria/ autos/2009/a004-09.htm

Hernández, R., Fernández, C. y Baptista, M. (2014). Metodología de la investigación. México: McGraw Hill.

Mavisoy, W. (2014). Etnografía sobre el quehacer antropológico y las manifestaciones de un antropólogo por su origen. El ejercicio de Jtsenojuabnayá «Existir reflexionando» Entre los kamëntšá. Tabula Rasa, 1(20),197-221.

Ministerio de Cultura. (s. f.). Caracterizaciones de los pueblos indígenas de Colombia. http://www.mincultura. gov.co/prensa/noticias/Documents/Poblaciones/ PUEBL0\%20EMBERA-D\%C3\%93BIDA.pdf 
Ministerio de Cultura. (2010). Embera Chamí. http:// observatorioetnicocecoin.org.co/cecoin/files/Caracterizaci\%C3\%B3n\%20del\%20pueblo\%20Embera $\% 20$ Cham\%C3\%AD.pdf

Ministerio de Educación Nacional (2015). Juegos infantiles $y$ aretes tradicionales del pueblo embera katío del alto Sinú. http://aprende.colombiaaprende.edu.co/sites/ default/files/naspublic/13_juegos_tradicionales.pdf

Moreno, G. (2008). Juego tradicional colombiano: una expresión lúdica y cultural para el desarrollo humano. Revista Educación Física y Deporte, 27(2), 93-99.

Rojas, A. y Castillo, E. (2005). Educar a los otros, Estado, políticas educativas y diferencia cultural en Colombia. Popayán: Universidad del Cauca.

Tamayo, C. y Cuellar, R. (2016). Juegos de lenguaje en movimiento: Una experiencia indígena. Revista Latinoamericana de Etnomatemática, 9(1), 49-70. http://www. revista.etnomatematica.org/index.php/RevLatEm/ article/view/238
Sánchez, I., Aguirre, W. y Ochoa, J. (2015). La identidad cultural como elemento clave para profundizar en los procesos educativos que apunten a la convivencia en sociedades multiculturales. Revista Praxis, 11(1), 61-75. http://dx.doi.org/10.21676/23897856.1554

Ulloa, E. (1992). Grupo indígena Los Emberá. Geografía Humana de Colombia. Región del Pacífico (tomo IX. Primera edición). Bogotá: Instituto Colombiano de Cultura Hispánica.

Vasco, L. (1987). Semejante a los dioses. Cerámica y cestería embera-Chamí. Bogotá: Universidad Nacional de Colombia. http://www.luguiva.net/admin/pdfs/Semejantes\%20a\%20los\%20dioses.pdf 